\title{
Lung cancer screening trials: Denmark and beyond
}

\author{
Jesper H. Pedersen, MD, DMsci, Rene H. Petersen, MD, and Henrik J. Hansen, MD
}

Lung cancer is the leading cause of cancer death worldwide, with fewer than $15 \%$ of patients surviving 5 years after diagnosis, despite advances in treatment during the past 20 years. Lung cancer screening (secondary prevention), in addition to smoking cessation programs (primary prevention), might be the most exciting improvement in methods to reduce lung cancer mortality. The great interest in lung cancer screening was started by the introduction of low-dose multidetector computed tomography (CT) in the 1990s. The nonrandomized International Early Lung Cancer Action Project study $(\mathrm{n}=32,000)^{1}$ was followed by several randomized clinical trials of high-risk current and former smokers. These included the National Lung Screening Trial (NLST) in the United States $(\mathrm{n}=53,500),{ }^{2}$ the DutchBelgian screening trial $(\mathrm{n}=15,822),{ }^{3}$ the Danish Lung Cancer Screening Trial (DLCST, $\mathrm{n}=4104$ ), ${ }^{4}$ and several other collaborating trials in Europe..$^{5-7}$ Recently, the National Cancer Institute published the report that the NLST gave evidence for a mortality reduction of $20.3 \%$ with low-dose CT screening compared with chest radiographic screening and a 7\% overall mortality reduction after 3 annual screening rounds and 8 years of follow-up. ${ }^{2}$ The NLST was a very well-performed randomized trial and has convincingly shown for the first time that CT screening, in high-risk subjects, can lead to a substantial reduction in lung cancer mortality and that the screening overall was not detrimental, because a reduction of $7 \%$ in general mortality was also observed. These are extremely important findings. The European trials have been smaller, but all have had a control group without any screening performed (usual care) and, therefore, potentially, the difference between the mortality in the CT and control arms could be more pronounced. In addition, the trials were started 2 to 3 years after the NLST and, therefore, have not yet performed a pooling of data and presented a combined mortality analysis. The Dutch-Belgian screening trial and the DLCST are expected to perform a final mortality analysis in 2015 but contemplated a preliminary pooling of data in 2011.

\footnotetext{
From the Department of Thoracic Surgery RT, Rigshospitalet, University of Copenhagen, Copenhagen, Denmark.

Disclosures: Authors have nothing to disclose with regard to commercial support. Presented at the 3rd International Minimally Invasive Thoracic Surgery Summit, Boston, Massachusetts, October 7-8, 2011.

Received for publication Oct 14, 2011; accepted for publication March 22, 2012; available ahead of print April 19, 2012.

Address for reprints: Henrik J. Hansen, MD, Department of Thoracic Surgery RT, Rigshospitalet, University of Copenhagen, Copenhagen, Denmark (E-mail: henrik.jessen.hansen@rh.regionh.dk).

J Thorac Cardiovasc Surg 2012;144:S7-8 $0022-5223 / \$ 36.00$

Copyright (c) 2012 by The American Association for Thoracic Surgery doi: $10.1016 /$ j.jtcvs.2012.03.066
}

The DLCST is a 5-year prospective randomized controlled screening trial evaluating the effect of annual CT screening for lung cancer. The overall design and baseline results were published in $2009 .^{4}$

\section{METHODS}

From October 2004 to March 2006, 4104 men and women were enrolled in the DLCST after recruitment by advertisements in local and regional free-of-charge newspapers, stating the purpose, study design, and inclusion criteria. The trial was funded in full by a governmental grant and was without costs to the participants.

The inclusion criteria were men $(55 \%)$ and women $(45 \%)$, aged 50 to 70 years, who were current or former smokers with at least 20 pack-years of smoking history. Former smokers should have quit for no more than 10 years. Lung function was measured by spirometry and forced expiratory volume in 1 second had to be at least $30 \%$ of predicted.

The exclusion criteria were a body weight greater than $130 \mathrm{~kg}$, a history of cancer diagnosis and treatment, lung tuberculosis, expected life expectancy of less than 10 years, and chest CT scan received during the past year for any reason.

Randomization was to either a screening group $(n=2052)$ or a control group $(\mathrm{n}=2052)$. The screening group received 5 annual, low-dose chest CT scans ( 1 baseline scan and 4 incidence scans). All came for an annual visit to the screening clinic, at which lung function tests were performed and questionnaires concerning health, lifestyle, smoking habits, and psychosocial consequences of screening were completed. ${ }^{1}$ The CT scans were evaluated by 2 experienced radiologists, ${ }^{1}$ with volumetric analysis of the detected nodules validated as in the Dutch-Belgian screening trial. ${ }^{8}$ Positron emission tomography scans were used to supplement the radiologic assesment. ${ }^{9}$

\section{RESULTS}

The DLCST showed that smoking habits during screening were unaffected by CT screening per se at 1 year of follow-up. ${ }^{10}$ Contamination by off-study CT scans in the control group was low $(0.04 \%){ }^{11}$

In the CT screening group, a total of 68 lung cancers were diagnosed. At baseline, the detection rate was $0.8 \%,{ }^{4}$ and the incidence rounds had a mean annual detection rate of $0.6 \%$ (chi-square test, $P=.492$ ). Of the 68 cases, 3 were small cell lung cancer (SCLC) and 65 were non-SCLC. Also, 57 (84\%) were early stage (stage I-IIIA non-SCLC and limited-stage SCLC) and thus potentially curable, and $11(16 \%)$ were diagnosed in a late stage (stage IIIB-IV non-SCLC and extensive-stage SCLC). One interval cancer was diagnosed 10 months after the third incidence scan.

Significantly more lung cancers were diagnosed in the screening group (chi-square test, 68 vs $24 ; P<.001$ ) and more were low stage (chi-square test, 57 vs $10 ; P<.001$ ). The number of late-stage lung cancers was the same in both groups (chi-square test, 11 vs $14 ; P=.640$ ). The number of new lung cancers remained high during all 4 incidence rounds in the screening group compared with 
the control group $(P<.001)$. A high proportion of the screen-detected lung cancers $(>75 \%)$ were treated by minimally invasive surgery (video-assisted thoracic surgery; data to be published).

\section{CONCLUSIONS}

The experience from the lung cancer CT screening trials have shown that CT screening will lead to increased detection of early-stage lung cancer and, consequently, an increased number of early-stage cases will be referred for surgical treatment. Most of these cases will be suitable for minimal invasive treatment. It is important that surgeons prepare themselves for this scenario, to deliver highquality minimally invasive surgery to their patients, whenever appropriate.

\section{References}

1. Henschke CI, Yankelevitz DF, Libby DM, Pasmantier MW, Smith JP, Miettinen OS, International Early Lung Cancer Action Program Investigators. Survival of patients with stage I lung cancer detected on CT screening. $N$ Engl J Med. 2006;355:1763-71.

2. The National Lung Screening Trial Research Team. Reduced lung-cancer mortality with low-dose computed tomographic screening. N Engl J Med. 2011;365:395-409.
3. van Klaveren RJ, Oudkerk M, Prokop M, Scholten ET, Nackaerts K, Vernhout R, et al. Management of lung nodules detected by volume CT scanning. $N$ Engl J Med. 2009;361:2221-9.

4. Pedersen JH, Ashraf H, Dirksen A, Bach K, Hansen H, Toennesen P, et al. The Danish randomized lung cancer CT screening trial-overall design and results of the prevalence round. J Thorac Oncol. 2009;4:608-14.

5. Lopez Pegna A, Picozzi G, Mascalchi M, Carrozzi FM, Carozzi L, Comin C, et al. Design, recruitment and baseline results of the ITALUNG trial for lung cancer screening with low-dose CT. Lung Cancer. 2009;64:34-40.

6. Infante M, Cavuto S, Lutman FR, Brambilla G, Chiesa G, Ceresoli G, et al. A randomized study of lung cancer screening with spiral computed tomography: three year results from the DANTE trial. Am J Respir Crit Care Med. 2009; 180:445-53.

7. Baldwin DR, Duffy SW, Wald NJ, Page R, Hansell DM, Field JK. UK Lung Screen (UKLS) nodule management protocol: modelling of a single screen randomised controlled trial of a low-dose CT screening for lung cancer. Thorax. 2011; 66:308-13.

8. Ashraf H, de Hoop B, Shaker SB, Dirksen A, Bach KS, Hansen H, et al. Lung nodule volumetry: segmentation algorithms within the same software package cannot be used interchangeably. Eur Radiol. 2010;20:1878-85.

9. Ashraf H, Dirksen A, Loft A, Bertelsen AK, Bach KS, Hansen H, et al. Combined use of positron emission tomography and volume doubling time in lung cancer screening with low-dose CT scanning. Thorax. 2011; $66: 315-9$.

10. Ashraf H, Tonnesen P, Pedersen JH, Dirksen A, Thorsen H, Dossing M. Smoking habits were unaffected by CT screening at 1 year follow-up in the Danish Lung Cancer Screening Trial (DLCST). Thorax. 2009;64:388-92.

11. Saghir Z, Ashraf H, Dirksen A, Brodersen J, Pedersen JH. Contamination during 4 years of annual CT screening in the Danish Lung Cancer Screening Trial (DLCST). Lung Cancer. 2011;71:323-7. 